\title{
Identifying fallacious arguments in a qualitative study of antipsychotic prescribing in dementia
}

Article

Accepted Version

Donyai, P. (2017) Identifying fallacious arguments in a qualitative study of antipsychotic prescribing in dementia. International Journal of Pharmacy Practice, 25 (5). pp. 379387. ISSN 0961-7671 doi: https://doi.org/10.1111/ijpp.12328 Available at https://centaur.reading.ac.uk/67982/

It is advisable to refer to the publisher's version if you intend to cite from the work. See Guidance on citing.

To link to this article DOI: http://dx.doi.org/10.1111/ijpp.12328

Publisher: Wiley InterScience

All outputs in CentAUR are protected by Intellectual Property Rights law, including copyright law. Copyright and IPR is retained by the creators or other copyright holders. Terms and conditions for use of this material are defined in the End User Agreement.

$\underline{\text { www.reading.ac.uk/centaur }}$

\section{CentAUR}


Central Archive at the University of Reading

Reading's research outputs online 


\section{Abstract:}

2 Background

3 Dementia can result in cognitive, non-cognitive and behavioural symptoms which are

4 difficult to manage. Formal guidelines for the care and management of dementia in the UK

5 state that antipsychotics should only be prescribed where fully justified. This is because

6 inappropriate use, particularly problematic in care home settings, can produce severe side-

7 effects including death. The aim of this study was to explore the use of fallacious arguments

8 in professionals' deliberations about antipsychotic prescribing in dementia in care-home

9 settings. Fallacious arguments have the potential to become unremarkable discourses that

10 construct and validate practices which are counter to guidelines.

\section{Methods}

12 This qualitative study involved interviews with 28 care-home managers and health

13 professionals involved in caring for patients with dementia. Potentially fallacious arguments

14 were identified using qualitative content analysis and a coding framework constructed from

15 existing explanatory models of fallacious reasoning.

\section{Key findings}

17 Fallacious arguments were identified in a range of explanations and reasons that participants gave in answer to questions about initiating, reducing doses of, and stopping antipsychotics in

19 dementia. The dominant fallacy was false dichotomy. Appeal to popularity, tradition,

20 consequence, emotion, or fear, and the slippery slope argument were also identified.

\section{Conclusions}


1 Fallacious arguments were often formulated to present convincing cases whereby prescribing

2 antipsychotics or maintaining existing doses (versus not starting medication or reducing the

3 dose, for example) appeared as the only acceptable decision but this is not always the case.

4 The findings could help health professionals to recognise and mitigate the effect of logic-

5 based errors in decisions about the prescribing of antipsychotics in dementia. 


\section{INTRODUCTION}

2 Dementia, which is characterised by an impairment of cognitive function, can also lead to

3 non-cognitive symptoms and behaviour that challenges carers and others. In the UK, the

4 National Institute for Health and Care Excellence (NICE) provides detailed guidance on the

5 care and treatment of dementia, including interventions for cognitive as well as non-cognitive

6 symptoms and behaviours [1]. The guidelines advocate that a range of non-pharmacological

7 interventions should be considered for those who develop non-cognitive symptoms or

8 behaviour that challenges and that pharmacological intervention (e.g. antipsychotics) should

9 be offered in the first instance only where patients are severely distressed or there is an

10 immediate risk of harm to the person or others, and only after a range of other conditions

11 have been met [1].

12 Yet it is estimated that only $20 \%$ of 180,000 patients with dementia who are prescribed an antipsychotic each year may actually benefit from taking these medications [2]. This was one of the findings of Professor Banerjee's landmark report which investigated the use of antipsychotics for people with dementia in the National Health Service in England [2].

Banerjee reported that inappropriate use could be resulting in an additional 1,620 cerebrovascular events and another 1,800 deaths each year. This was despite existing warnings by various medicines regulators about the use of antipsychotics in dementia [3-5]. Since then, several national studies and audits have shown a general downward trend in antipsychotics prescribing in dementia using information from primary care clinical systems [6] and hospitals [7-9]. But these data do not reveal the prescribing pattern in care homes where a third of UK patients with dementia are estimated to reside [10]. Care homes are accommodation that provide 24-hour nursing care (i.e. nursing homes), personal care only (i.e. residential homes) or a combination of both to older people in the UK. 
1 A qualitative study with old age psychiatrists exploring the prescribing of psychotropic

2 medication in dementia uncovered a range of views [11]. Psychiatrists thought there were

3 pressures on them to prescribe, felt societal and systemic influences maintained high

4 prescribing rates, guidelines were not implementable, and care homes were not designed and

5 staff not trained to deal with problematic behaviours. While some of these views may be

6 valid, problematic arguments relating to antipsychotic prescribing in dementia, especially if

7 they become everyday discourses, are potentially significant as they could reinforce poor

8 practice, but this concept has not been formally investigated. The premise of this paper is that

9 reasoning errors could have a role in constructing and validating antipsychotic prescribing in

10 dementia in practice akin to what has been argued to occur in attention deficit hyperactivity

11 disorder (ADHD) by Tait [12]. Reasoning errors in this context are invalid or faulty explanations used in a discussion resulting in erroneous or fallacious arguments. Tait studied the contribution of fallacious reasoning in reinforcing the veracity of ADHD as a mental health condition [12]. Using the typology set out by Fearnside and Holther (cited in [12]) of material, psychological, logical fallacies, Tait presented numerous examples to illustrate the way in which fallacious arguments potentially verify the existence of ADHD and therefore a need for its treatment. The diagnosis and treatment of ADHD has been brought into question by others $[13,14]$ but Tait's position was that logic-based errors could influence everyday medical practice - fallacious arguments about ADHD become unremarkable discourses that construct and validate ADHD as a treatable disorder leading to prescribing where it might not be warranted. The case being made was that fallacious arguments potentially endorse inappropriate prescribing.

The claim that fallacious arguments could underline potentially inappropriate prescribing is novel and worthy of further consideration. The aim of this paper is to explore professionals' deliberations about antipsychotic prescribing in dementia for evidence of fallacious 
1 arguments using qualitative content analysis [15] and a constructivist approach [16]. The

2 research question was "Do health professionals and care-home managers use fallacious

3 arguments in discussions about antipsychotic prescribing in dementia and if so, what is the

4 nature of these?"

\section{METHODS}

\section{Compliance with Ethical Standards}

7 The University's Research Ethics Committee (UREC 1217), and the local NHS Research \&

8 Development office (letter of access granted 22/06/2012) and Primary Care Research

9 Partnership (reference TV85) reviewed and approved the research. Written consent from each participant was obtained before the interviews.

\section{Design}

Professionals with a role in the care and management of patients with dementia in care homes were recruited to the study using purposive sampling to select primary- and secondary-care doctors, care-home managers, primary-care pharmacists, and community psychiatric nurses. A memory clinic nurse and a social worker were later recruited on the recommendation of existing interviewees. Recruitment was through posted letters using publicly-available addresses or through known contacts and already recruited interviewees. In-depth semistructured face-to-face interviews were carried out by a doctoral student, supervised by the author, using interview schedules that focused first on general descriptions of dementia and the progression of this condition, before considering beliefs about and professional experiences with antipsychotic prescribing in dementia. Interview schedules were piloted with three volunteer participants before use. The interviews were audio-recorded. Participants were recruited until no more new codes and concepts emerged to inform the study (i.e. sampling saturation). 
1

2 The interviews were transcribed verbatim into password-protected documents, removing

certain information to keep data anonymised/de-identifiable. The doctoral student ensured

4

5 from the explanatory model described by Tait [12] and other published sources [17,18]

6 (Table 1). The qualitative content analysis was undertaken by a consortium of six Masters-

7 level students of pharmacy who worked with the author to learn the analytical process during

8 a 3-month training period preceding data analysis. Each student was asked to individually

9 analyse the same sample section of interview transcript with reference to Table 1. Group meetings allowed students to compare and contrast the coding and discuss and resolve any differences to reach consensus on the analytical approach.

After familiarization with each transcript, the text was examined line-by-line to identify potentially fallacious arguments with reference to Table 1 . The sentences and phrases appearing to exhibit fallacious reasoning were all labelled. Then during a second coding phase each initial code was examined in more detail to delineate the elements that were indicative of an erroneous argument. Each valid example was categorised according to the type of fallacy it revealed. The group collaboratively considered and reached consensus on the coding of each excerpt with the author's direct involvement. Cases were grouped according to fallacy type. Data validation was demonstrated in data triangulation (collecting data from 8 participant sub-groups), description of the study procedures, and audit trails.

\section{Researcher characteristics and reflexivity}

A Saudi pharmacist and doctoral student completed the interviews. His status as a 'non-UKnational', thus lack of UK experience, empowered him to ask impartial questions where the author, a UK-based health professional and academic might have been at a disadvantage due to her professional involvement. Nevertheless, the author's dual qualifications (as a 
1 pharmacist and a psychologist) were valuable in bridging the clinical (antipsychotic

2 prescribing) and investigative (fallacious reasoning) domains during analysis, and involving

3 six Masters-level pharmacy students increased trustworthiness through group review.

\section{RESULTS}

5 A total of 28 participants (17 female) were interviewed from May 2012 to February 2013.

6 The sample included care-home managers $(\mathrm{CHM})(\mathrm{n}=5)$, general practitioners $(\mathrm{GPs})(\mathrm{n}=5)$,

7 community psychiatric nurses $(\mathrm{CPNs})(\mathrm{n}=7)$, psychiatrists $(\mathrm{n}=5)$, geriatricians $(\mathrm{n}=2)$,

8 primary-care pharmacists $(n=2)$, a memory-clinic nurse, and a social worker.

9 The dominant, recurring fallacy was the false dichotomy fallacy (around a third of the

10 recurring fallacies) and there were also examples of appeal to popularity, tradition,

11 consequence, emotion or fear (with the 'appeal to' fallacies accounting for around half of the examples), and the slippery slope argument (around a fifth of the cases). These examples spanned the categories set out by the typology of Fearnside and Holther (cited in [12]) and occurred mainly in answers to questions about initiating, reducing the dose of, and stopping antipsychotics.

\section{False dichotomy}

17 Fearnside and Holther (cited in [12]) considered false dichotomy to be a material fallacy, meaning the material of an argument is poorly prepared with an incorrect conclusion being drawn ${ }^{1}$. False dichotomy was adopted by a range of respondents in relation to the use of antipsychotics in patients with dementia. For examples, see the following response to "On balance do the benefits of medication for behavioural difficulties outweigh the concerns?"

\footnotetext{
${ }^{1}$ False dichotomy is where choice is limited to one of two alternatives without highlighting other potentially viable options.
} 
"So in some people's cases it, it's the lesser of two evils. You don't want to give them medication but you don't want them to, the whole home situation to fall apart yeah so it's weighing it up...." (CPN 11).

As one of the options is particularly undesirable, maintaining the patient on a high dose is not only logical (as the argument is presented) but necessary. A range of professionals made similar arguments, presenting the choice as that of an antipsychotic being initiated or maintained versus severe or unwanted disruption of care - and no mention of outcomes between the two extremes. In giving these responses, the interviewees neglected to present other options in their argument, such as a trial reduction of the dose of an antipsychotic, or non-pharmacological approaches for addressing symptoms.

A different false dichotomy argument is illustrated in a psychiatrist's description of a typical care-home setting in the absence or presence of antipsychotic medication. The following is a response given to "What's your opinion in general about guidelines that relate to prescribing of antipsychotics in dementia?"

"....So they continue on these antipsychotics for ever and ever, they go into nursing homes, in nursing homes given a choice between having somebody who's going to wander around or somebody's who's going to be fairly quiet, sleep in a chair through their shift, staff will always choose to have somebody who's going to be quiet and sleep in their chair because it makes for much easier shifts for them. So they never want anyone's antipsychotics stopped and I think this is part of the problem. And I think the guidelines aren't very clear about what needs to happen." (Psych 14)

The complex situation in a care home is distilled down and presented as two simple alternatives, with one clearly an emotive and controversial opinion about the management of care homes. Another type of false dichotomy seen in the transcripts is exemplified in a GP's response to the same question as above; in the response, a stark contrast is drawn between using guidelines and 'going to the other extreme' and not prescribing them: 
"I don't think, I'm not very familiar with the guidelines. I think, but what I know of them, I think we have to be careful not to go to the other extreme where we just say we're not prescribing them." (GP 23)

\section{Appeal to popularity/tradition/consequence/emotion/fear}

Certain types of fallacies can play up the rhetorical element of an argument, as a tactic to win the listener over. These were considered by Fearnside and Holther (cited in [12]) to be psychological fallacies, meaning that the speaker makes a slip-up or uses a 'trick' while presenting the argument - i.e. does not use correct evidence to back the argument.

Appeal to popularity was used numerously in relation to the perceived role of the media in directing practice. An example is the response provided by a CPN when asked "And would you tell me please about when should antipsychotics be given to patients with dementia?"

"When? I mean you see a lot of stuff in the news that obviously, the health risks of giving antipsychotics to people with dementia and they should be avoided whenever possible." (CPN 12)

The response below from an interview with a GP is another example of appeal to popularity.

It is given in relation to "And do you think there's any change in prescribing antipsychotics in the recent years, that's different from the past?"
"There is such a big group of patients now who are all, antipsychotics are tried for so I think the use of them is increasing maybe because, I don't know, we are less afraid of them. I think doctors are less afraid of using antipsychotics which wasn't the case before so I would do a prescription for risperidone if I get told, right, increase the dose or can we titrate the dose? Right, fine. I'll call the patient in every month, gradually increase the dose without worrying." (GP 28).

Here, the validity of the argument to prescribe antipsychotics appears to rest on a sense that because there is 'such a big group of patients' for whom antipsychotics are tried that this is acceptable practice. No evidence is offered for being 'less afraid' or not 'worrying' about antipsychotic prescribing other than the high occurrence of their use 
1 Appeal to tradition was also used and one example is the response from a GP interview to

2 "And do you think prescribing antipsychotics for behavioural difficulties in dementia are

3 always justified?"

"They should always be justified but I think it's still used as the easy option. Because it's something as doctors, we do, we just prescribe a medicine." (GP 23)

Here, the validity of what happens in practice appears to rest on the fact that doctors 'just prescribe a medicine' (which may well be the traditional role of doctors); but prescribing may not always be best for the patient.

Appeals to consequence, emotion or fear were also identified. Note for example the way in which a CPN appeals to the consequence of not giving an antipsychotic to a patient with dementia, from the perspective of carers in response to: "To what extent do you think that patients and their relatives can be practically made aware of reasons why antipsychotics are prescribed?"
"Well I think when we assess them we tell them, we probably tell them verbally. I'm not sure what information they would then get from Pharmacy. But we always tell people about the risks as well as the benefits and I think most people say, I'm willing to take that risk because this is not, I can't cope with this at the level it's at so yeah." (CPN 11)

Here, the natural conclusion is therefore to give the antipsychotic because the consequence otherwise is that the carer would not 'cope' - this outcome is undesirable and also plays on the emotional aspects of the situation. Some of the examples of 'false dichotomy' also contained appeals to consequence, emotion or fear.

This multiplicity is illustrated in the next example which relates to an instance where the dose of an antipsychotic could potentially have been reduced. The question was "And what about the feedback from the relatives after prescribing antipsychotics?" 
"So I come back and fed that back to her doctor and the doctor said, well we have to respect the fact that he's doing a very, very hard job keeping her at home and home is where she wants to be, home is where he wants her to be." (CPN 11)

Here, the clinical decisions made by health professionals are presented in the context of a desire to keep the patient at home. An appeal is made to the consequence of reducing the antipsychotic dose, which in this case would mean the patient being unable to stay at home there is also an appeal to emotion as the patient's husband has been doing a 'hard job keeping her at home' and would doubtless be distraught to see her leave. All at the same time, a false dichotomy is created by presenting only two alternatives - being able to stay at home (if dose maintained) or having to leave. The same type of argument is made by a psychiatrist in relation to stopping mediation in response to: "And are there any, is there an improvement after that medication has been prescribed?"
"Yes. Oh definitely, yes. So it makes a huge difference and you'll be surprised by the number of times the families actually say to me it's made such a huge difference. And they are the ones who say no, no don't stop the antipsychotic because we know how different things were before they were on them because they see that level of distress reducing in those patients with the antipsychotics." (Psych 14)

Appeals to consequence, emotion or fear were not limited to the (positive) impact of prescribing antipsychotics on carers. A proportion of examples related to the impact of prescribing on patients' own state. The example below illustrates the perceived effect of antipsychotics on patients in response to: "And how long does it take for this benefit [of prescribing antipsychotics] to show?"

"Initially a couple of days because their, it gets into their system and I, it appears that it makes them a little bit more sleepy until their body adjusts to it so they are calmer, but as they get used to it in their system we notice that they're just more co-operative and more relaxed." (CHM 3)

An additional example is given here in response to: "And what are the benefits [of antipsychotic prescribing]?” 
"They're quieter, more subdued, less distressed. The man that I saw last week that had been prescribed them, who's got dementia, he was just weeping inconsolably, he's got dementia and he was weeping for his wife who has been dead for many years, and they put him on quetiapine. And I mean he gets tearful if you talk about his wife now, but the uncontrollable weeping had stopped." (GP 7)

\section{Slippery slope argument}

The slippery slope argument was considered by Fearnside and Holther (cited in [12]) to be a logical fallacy, meaning that the machinery of the argument malfunctions - i.e. there is a structural breakdown in the logic of what is presented. The example provided below was typical, provided in response to: "On balance do the benefits of medication for behavioural difficulties outweigh the concerns?"

"It's funny I was just talking to one of our consultants about a lady that I visit, if she wasn't on these, the medication, at the level she's on, her husband wouldn't be able to manage her at home. But I think if he couldn't manage at home then she would have to go into residential care and if she was in residential care they would need far higher doses of the medication to manage her." (CPN 11)

The excerpt focusses on antipsychotic usage in a patient with dementia living at home, as described by a CPN. Again, there are several other fallacies also in operation here. The slippery slope element relates to the chain of events predicted to happen should the patient not have been on 'the medication, at the level she's on'. A prediction of gloom is made 'she would have to go into residential care and if she was in residential care they would need far higher doses of the medication to manage her' whereas in actual fact reducing the dose might not have resulted in a) the husband not being able to cope, b) the patient leaving their own home and going into residential care, c) care-home staff not being able to manage the patient, d) patient therefore needing higher doses. 


\section{DISCUSSION}

2 A range of fallacious arguments with the potential to incorrectly construct and validate antipsychotic prescribing in dementia were identified in health professional and care-home manager interviews. False dichotomy was the dominant fallacy and also appeal to popularity, tradition, consequence, emotion or fear, and the slippery slope argument were identified in the interviewees' answers to questions about antipsychotic prescribing in dementia. False dichotomy was frequently used to explain the prescribing of antipsychotics or the continuation of an already-prescribed dose. This type of argument can be particularly convincing when one of the choices is framed in such bad light that the other seems the only viable option. The arguments were formulated to illustrate the negative consequences of alternative choices (e.g. reducing the dose or not prescribing) on carers and patients, for example in terms of coping and stress; whether the patient could remain in their own home or be manageable within a care-home setting. False dichotomies can be seen not to really present a choice to the listener. They are framed such that not prescribing antipsychotics, actually following the guidelines, or lowering the dose of antipsychotics, seem detrimental to patient care.

Sometimes false dichotomies were supplemented with the negative aftereffects framed as a series of 'slippery slope' events, again presenting the situation in such a way that prescribing or maintaining the dose of an already-prescribed antipsychotic became the only apparent option. Appeal to popularity was also identified, communicating the perceived authority of the media in driving practitioner behaviours. Yet the truth should not emerge from the news and health professionals have a duty to base prescribing decisions on published guidelines and other published evidence. Appeal to popularity as a form of fallacious argument was also used to justify the prevalence of antipsychotic prescribing in dementia, from a 'safety in numbers' standpoint. Doctors may be justified or not justified in prescribing antipsychotics 
1 but there is no safety in numbers and being part of a large group does not reduce the risks.

2 Similarly, appeal to tradition was used to support existing practices relating to antipsychotic

3 prescribing or review as were appeals to consequence, emotion or fear.

4 Taken at face value appeals to consequence, emotion or fear, are extremely powerful

5 arguments because they manipulate the listener's emotions to make a convincing argument.

6 The point is that (according to the NICE guidelines [1]) antipsychotics should not be

7 prescribed to reduce stress in carers, enable carers and care-home staff to cope, keep the

8 patient at home, allow the patient to attend a day centre, reduce stress in carers, or because

9 the carer is crying. Nor should they be prescribed to make the patient cooperative, relaxed,

10 calm, quiet, subdued, because they were distressed, weeping or agitated. These are not

11 indications listed in the marketing authorisation for antipsychotics.

12 Fallacies in care-home managers' and health professionals' discussions about antipsychotic prescribing in dementia were identified among those based in one English county, although the sample size is in line with other qualitative studies utilizing in-depth interviews. How people think and speak communicates and corroborates their understanding of social phenomena; it also has a role in constructing and verifying their version of reality, which in turn has the potential to impact on their own, and others' actions and behaviours [16]. The fallacious arguments in the conversations appeared to authenticate potentially inappropriate prescribing and could in theory contribute to the practice through implicit assumptions about these medicines that could shape opinions and therefore actual practice. But the examples of fallacious reasoning in themselves do not provide direct evidence for inappropriate practice; they are responses provided in good faith to interview questions. So it is essential to state that while this paper focusses on the fallacious arguments made by the participants, this is not to establish bad practice on their part, but to illustrate the types of arguments that can be made by professionals in general. 
1 A recent US study found that the reasons given for the use of antipsychotic medication in

2 nursing-home residents with dementia frequently related to a wide variety of indications for

3 which the medications are not approved and for which evidence of efficacy is lacking [19].

4 For example, as well as psychiatric (e.g. loss of contact with reality, depression, anxiety) and

5 behavioural reasons (e.g. verbal and physical aggression), emotional reasons were cited for

6 the use of antipsychotics including that the resident was angry or agitated, or even "sad" or

7 “crying" - thus also linking emotional consequences to inappropriate prescribing [19].

8 Numerous fallacious arguments in this study concerned coping with the patient, either within

9 their own home environment or within a care-home setting and it was not possible to draw a distinction between the two settings in terms of the arguments used although there are numerous studies that demonstrate higher antipsychotic use in formal care settings. For example, a study published in 2012 reported that while $7.3 \%$ of people with dementia living in their own home received an antipsychotic prescription, this compared to $25.5 \%$ of patients with dementia in care homes [20]. In another study published in 2013, psychotropic medication use in general was found to be higher in care homes compared to the community setting $(20.3 \%$ vs $1.1 \%)$, and antipsychotics prescribing increased from $8.2 \%$ before entry to $18.6 \%$ after patients entered care homes [21]. These differences could of course be associated with an increase in symptom severity as the patient moves from their own home to the care home environment.

International studies draw attention to variability in the use of antipsychotics between different care homes, which authors relate to care home characteristics and patient satisfaction [22] and characteristics of psychiatric consultant groups [23]. Certainly a multitude of interventional studies have attempted to reduce antipsychotic prescribing for people with dementia in care homes, and while some interventions are effective in the shortterm, there is a continuing need for effective interventions that might address the culture and 
1 nature of the different care settings [24]. Identifying a role for fallacious arguments in

2 potentially inappropriate antipsychotic prescribing in dementia can inform future studies that

3 focus, for example, on changing people's thinking patterns and reasoning. The categorisation

4 of fallacious arguments as material, psychological and logical fallacies may be helpful in

5 such studies, which could focus on highlighting and challenging the common arguments that

6 construct and validate antipsychotic prescribing and continuation of prescribing in dementia,

7 where it is not warranted.

\section{CONCLUSIONS}

9 This is the first study that examines in depth the use of fallacious arguments in relation to initiating, reducing the dose of and stopping antipsychotics in dementia. Through false dichotomy in the main and also slippery slope argument and appeal to a range of conditions, the case presented in this article is that fallacious arguments used by professionals involved in caring for patients with dementia could be constructing and validating implicit assumptions about antipsychotic prescribing in this condition. Where fallacious arguments are used, the rationale for not prescribing or for reducing the dose of antipsychotics already prescribed are convincingly presented in such undesirable light that prescribing or maintaining an existing dose become the only viable options. These types of fallacies are powerful and could sway practice. The findings could help practitioners, researchers and policy makers to contemplate and attempt to mitigate the effect of possible logic-based errors in the inappropriate prescribing of antipsychotics in dementia through formal training and interventions.

\section{References} dementia and their carers in health and social care. NICE guidelines [CG42]. November 
1 2006, Last updated: May 2016. [Online]. Available: https://www.nice.org.uk/guidance/CG42/

2 [Accessed 10 August 2016]

3 2. Banerjee S. The use of antipsychotic medication for people with dementia: Time for action.

4 London: Department of Health 2009.

5 3. U.S. Food and Drug Administration. Information for Healthcare Professionals:

6 Conventional Antipsychotics. June 2008. [Online]. Available:

7 http://www.fda.gov/Drugs/DrugSafety/PostmarketDrugSafetyInformationforPatientsandProvi

8 ders/ucm124830.htm. [Accessed 2 May 2015].

9 4. Medicines \& Healthcare Products Regulatory Agency. Antipsychotic medicines: licensed 10 products, uses and side effects. August 2005. [Online]. Available:

11 https://www.gov.uk/government/publications/antipsychotic-medicines-licensed-productsuses-and-side-effects. [Accessed 2 May 2015].

5. European Medicines Agency. CHMP assessment report on conventional antipsychotics. London: European Medicines Agency 2008.

6. Health and Social Care Information Centre. National Dementia \& Antipsychotic Prescribing Audit. Leeds: Clinical Audit Support Unit 2012.

7. Stephens P, Chikh K, Leufkens H. Prescribing of antipsychotics in people with dementia in acute general hospitals in England: 2010-2012. Eur Geriatr Med 2014;5:394-398.

8. Royal College of Psychiatrists. Report of the National Audit of Dementia Care in General Hospitals. London: Healthcare Quality Improvement Partnerships 2011.

9. Royal College of Psychiatrists. National Audit of Dementia care in general hospitals 201213: Second round audit report and update. London: Healthcare Quality Improvement Partnerships 2013.

10. Luengo-Fernandez R, Leal J, Gray A. Dementia 2010. The prevalence, economic cost and research funding of dementia compared with other major diseases. A report produced by the Health Economics Research Centre, University of Oxford for the Alzheimer's Research Trust. Cambridge: Alzheimer's Research Trust 2010. medications by old age psychiatrists for behavioural and psychological symptoms of dementia: a qualitative study. Age Ageing 2008;37:547-552. and Education 2009;28:239-254. everyday language. Anthropol Educ Q 2001;32:167-190. 
1 14. Conrad P, Potter D. From hyperactive children to ADHD adults: observations on the

2 expansion of medical categories. Social Problems 2000;47:559-582.

3 15. HsiehH-F, Shannon SE. Three Approaches to Qualitative Content Analysis. Qualitative

$4 \quad$ Health Research 2005;15: 1277-1288

5 16. Burr V. Social constructionism. 2nd ed. Hove: Routledge 2003.

6 17. Hofmann B. Fallacies in the arguments for new technology: the case of proton therapy. $J$

7 Med Ethics 2009;35:684-687.

8 18. Gibbs N. Formal and informal fallacies in anaesthesia. Anaesth Intensive Care

9 2010;38:639-646.

10 19. Bonner AF, Field TS, Lemay CA, et al. Rationales that providers and family members

11 cited for the use of antipsychotic medications in nursing home residents with dementia. J Am

12 Geriatr Soc 2015;63: 302-308.

13 20. Child A, Clarke A, Fox C et al. A pharmacy led program to review anti-psychotic

14 prescribing for people with dementia. BMC Psychiatry 2012;12:155.

15 21. Maguire A, Hughes C, Cardwell C, et al. Psychotropic medications and the transition into 16 care: a national data linkage study. J Am Soc Geriatr Soc 2013;61:215-21.

17 22. Kleijer B, van Marum R, Frijters D, et al. Variability between nursing homes in 18 prevalence of antipsychotic use in patients with dementia. Int Psychogeriatr 2014;26:363-71. 23. Tjia J, Field T, Lemay C, et al. Antipsychotic use in nursing homes varies by psychiatric consultant. Med Care 2014;52:267-71.

24. Coon J, Abbott R, Rogers M, et al. Interventions to Reduce Inappropriate Prescribing of Antipsychotic Medications in People With Dementia Resident in Care Homes: A Systematic Review. The Journal of Post-Acute and Long-Term Care Medicines 2000;15:706-718. 


$\begin{array}{lll}\text { Type of Fallacy } & \begin{array}{l}\text { Definition of Fallacy } \\ \text { Ad hominem [18] }\end{array} & \text { Form of Fallacy } \\ & \begin{array}{l}\text { A person's argument is based on their } \\ \text { presumed honesty, ethical character or }\end{array} & \text { Person A makes claim C. } \\ \text { trustworthiness. } & \text { Person B makes an attack on person A. } \\ \text { Ad novitam [17] } & \begin{array}{l}\text { It is assumed that the 'new' technology is } \\ \text { better than the existing technology and that it }\end{array} & \text { A is a new technology } \\ & \text { should be implemented and used. } & \text { A should be implemented and used. }\end{array}$

$\begin{array}{lll}\text { Affirming the } & \text { Inferring the truth of the antecedent of an } & \text { If A then B } \\ \text { consequent [12] } & \text { implication from the truth of the consequent. } & \text { B } \\ & & \text { So, A } \\ \text { Appeal to consequences } & \text { The argument concludes a premise (usually a } & \text { If you don't accept A as true, something } \\ \text { or to fear [18] } & \text { belief) is either true or false based on } & \text { terrible will happen. } \\ & \text { whether it leads to desirable or undesirable } & \text { Therefore, A must be accepted. }\end{array}$

$\begin{array}{llll}\text { Appeal to emotion [18] } & \text { Emotion is used in place of reason in an } & \text { Favourable emotions are associated with } \\ & \text { attempt to win the argument. } & \text { A. } & \text { Therefore, A is to be accepted. } \\ \text { Appeal to popularity (or } & \text { Peer-pressure or popularity is substituted for } & \text { A believes } \mathrm{X} \\ \text { bandwagon or Ad- } & \text { evidence in an argument. } & \text { B believes X } \\ \text { Populum fallacy) [12] } & \begin{array}{lll}\text { It is assumed that something is better or } \\ \text { Appeal to tradition [18] }\end{array} & \text { A is old or traditional } \\ \text { Begging the question } & \text { The conclusion of an argument is used as a } & \text { A implies B and A is only valid because } \\ \text { [12] } & \text { premise of that same argument. } & \text { Therefore, A is correct or better. }\end{array}$

(or circular reasoning)

$\begin{array}{lll}\text { Confirmation bias [18] } & \begin{array}{l}\text { Information is favoured that confirms } \\ \text { preconceptions or hypotheses regardless of }\end{array} & \text { A is claimed to be correct, because it } \\ & \text { whether the information is true. }\end{array}$




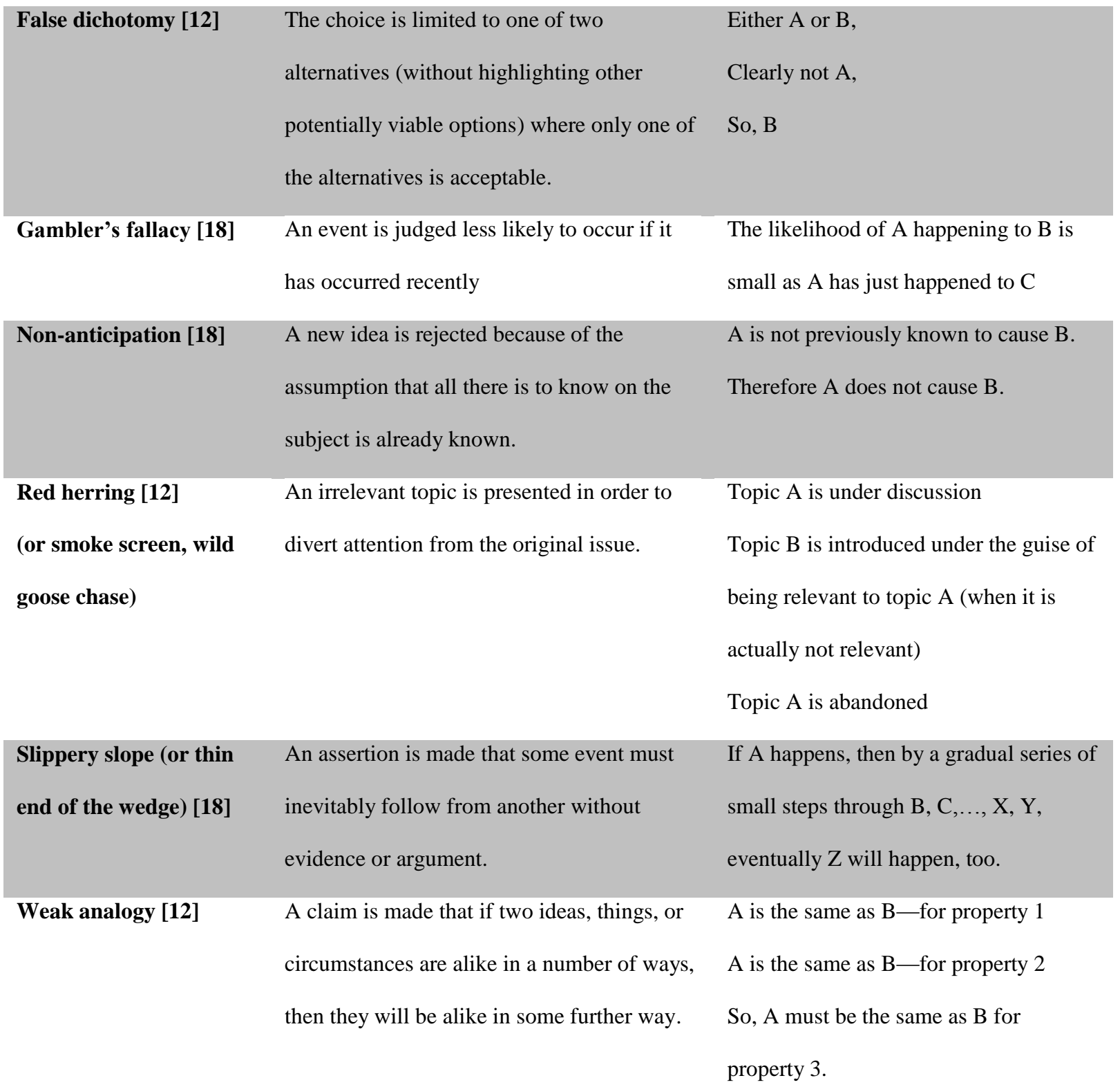

1 Table 1. Common fallacious arguments derived from the literature 